\title{
Concise Chemoenzymatic Total Synthesis of GE81112 B1 and Sim- plified Analogs Enable Elucidation of Its Key Pharmacophores
}

\author{
Christian R. Zwick III, Max B. Sosa, Hans Renata*. \\ Department of Chemistry, The Scripps Research Institute, 130 Scripps Way, Jupiter, FL 33458
}

\section{Supporting Information Placeholder}

ABSTRACT: The GE81112 complex has garnered much interest due to its broad antimicrobial properties and unique ability to inhibit bacterial translation initiation. Herein we report the use of a chemoenzymatic strategy to complete the first total synthesis of GE81112 B1. By pairing hydroxylase enzymes found in GE81112 biosynthesis with traditional synthetic methodology, we were able to access the natural product in 11 steps (longest linear sequence). Following this strategy, six GE81112 B1 analogs were synthesized, allowing for identification of its key pharmacophores.

GE81112 is a complex of three related tetrapeptides identified in 2006 during a screen for new inhibitors of bacterial protein synthesis (Figure 1A). ${ }^{1}$ Structurally, GE81112 consists of several unusual amino acids, including 3-hydroxy-L-pipecolic acid (AA1), 4-hydroxy-L-citrulline or O-carbamoyl-2amino-dihydroxyvaleric acid (AA2), 2-amino-L-histidine (AA3) and $\beta$-hydroxy-2-chloro-L-histidine (AA4). Each GE81112 tetrapeptide demonstrates broad antimicrobial properties conveyed by a unique mechanism of action: inhibition of bacterial translation initiation. ${ }^{1}$ Subsequent studies have confirmed that GE81112 binds to the 30S ribosome, inhibiting the P-site decoding of an mRNA's initiation codon by fMet-tRNA's anticodon. ${ }^{2}$ Although GE81112 displays broad-spectrum activity in minimal media, its activity is significantly attenuated against bacteria grown in rich media. ${ }^{1}$ Subsequent mechanistic studies indicate that illicit transport of GE81112 into its bacterial target is mediated by peptidyl transporter oligopeptide permease (Opp), but competition with peptides found in rich media results in reduced efficacy. ${ }^{3}$ Based on these findings, development of GE81112 analogs that can permeate the bacterial cell membrane without the aid of Opp presents a potential solution. To date, there is only one reported total synthesis of the GE81112s (GE81112 A, 2018) though minimal structure-activity relationship (SAR) has been established for this tetrapeptide. ${ }^{4}$ Additionally, the reported synthesis suffers from high stepcount (7-8 steps per fragment) and poor stereocontrol (Figure 1A). In light of these shortcomings, we sought to develop a tractable chemoenzymatic synthesis of GE81112 B1 that will also be amenable for analog generation for SAR analysis. 


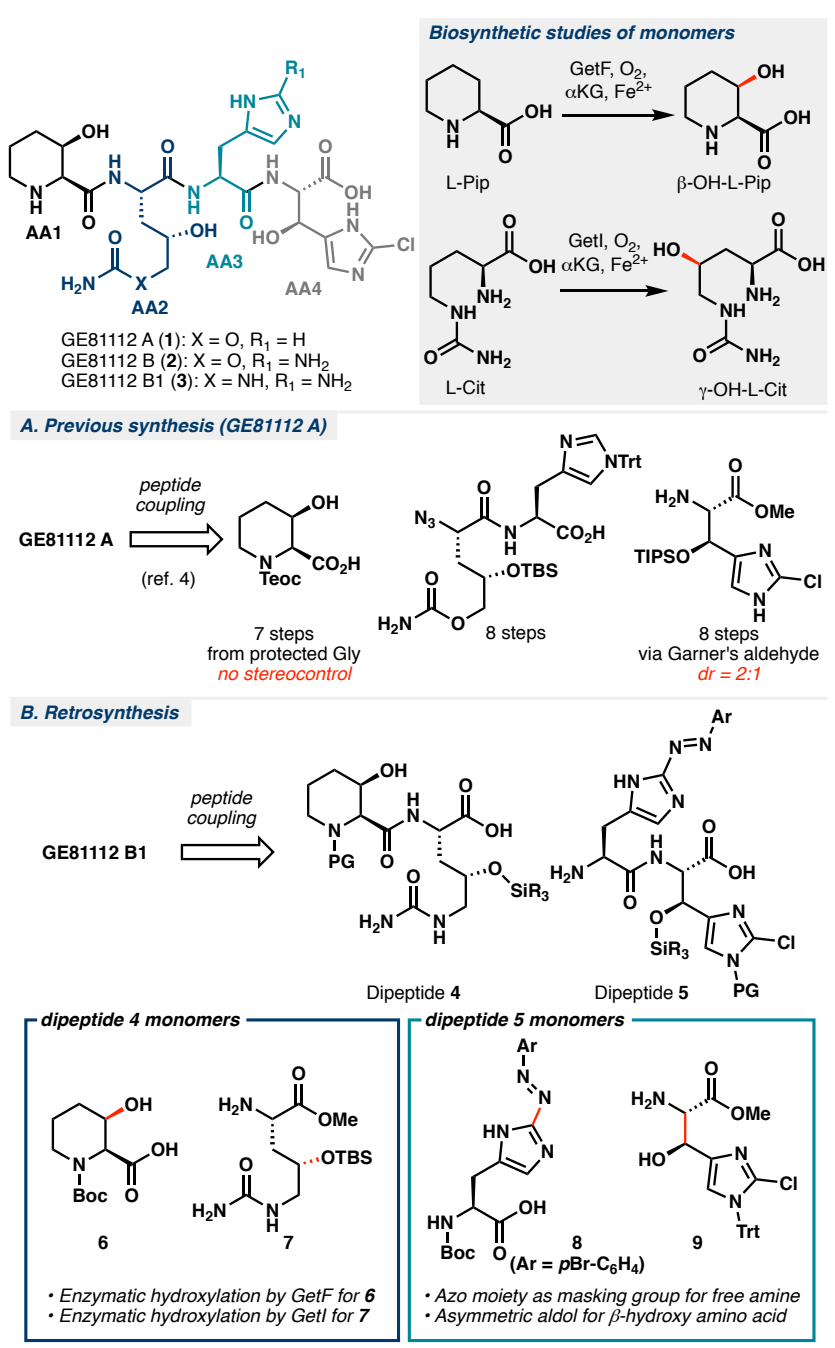

Figure 1. Structures of the GE81112 tetrapeptide complex. A. Known biosynthesis of its monomers. B. Our retrosynthetic analysis of GE81112 B1 (3).

Our synthetic design combines biocatalytic and contemporary chemical approaches to construct each fragment with minimal step count. ${ }^{5}$ To balance these synthetic paradigms, we devised a biocatalytic approach for the preparation of AA1 \& AA2, and traditional chemical approach for AA3 \& AA4. We envisioned utilizing $\mathrm{C}-\mathrm{H}$ hydroxylation reactions present in GE81112 biosynthesis to construct AA1 \& AA2 (Figure 1B). Previous reports established GetF and Getl as iron- and a-ketoglutarate dependent dioxygenase enzymes (Fe/aKG) responsible for hydroxylation of L-pipecolic acid (L-Pip) and L-citrulline (L-
Cit), respectively (Figure 1B). ${ }^{6}$ Next, a chemoselective azo coupling would establish a "masked" 2-aminoimidazole motif present within $A A 3$, aiding purification and imparting chemoselectivity in subsequent transformations. ${ }^{7}$ An asymmetric aldol transform would establish AA4 while also controlling the syn1,2-amino alcohol stereochemistry. Convergent assembly of each fragment, followed by protecting group removal would afford GE81112 B1. This strategy was also designed with modularity in mind, as further analogs of GE81112 B1 could be prepared by tapping into the wealth of sequence diversity of Fe/aKG hydroxylases for preparing alternative $A A 1$ and $A A 2$ units and using alternative aldehyde partners for preparing alternative AA4 units. While seemingly straightforward, successful implementation of our strategy would still require careful choreography of protecting group manipulations and condition selections to avoid chemoselectivity issues. For example, late-stage hydrogenative unmasking of the 2-azoimidazole functionality in the presence of reduction-prone 2-chloro-imidazole could present a major concern. Here, we rationalized that introduction of a suitable protecting group on the 2-chloroimidazole unit would allow for chemoselective azo hydrogenation based on differing steric environments.

We first sought to establish GetF and Getl as robust biocatalysts for the preparation of monomers AA1 and AA2. GetF was recently used to hydroxylate 3-methylproline in the synthesis of 3-hydroxy3-methylproline, although a large-scale reaction with L-Pip has not been reported. ${ }^{8}$ GetF was initially found to suffer from poor soluble expression when expressed as $\mathrm{N}-\mathrm{His}_{6}$-tagged protein, but co-expression of chaperones GroES/GroEL resulted in a dramatic improvement in soluble enzyme expression. ${ }^{8,9}$ For large-scale reactions, L-Pip was combined with 
aKG, $\mathrm{FeSO}_{4}$, ascorbic acid (asc) and lysate of $E$. coli BL21(DE3) co-expressing GetF and GroES/GroEL chaperone proteins. Complete conversion on 500 mg scale was possible utilizing $20 \mathrm{mM}$ substrate concentration, $\mathrm{pH}=8 \mathrm{kPi}$ buffer and clarified lysate (Scheme 1A, Table SI1). Following ion-exchange purification, the resulting crude material was converted to the corresponding N-Boc derivative, affording 6 in 74\% yield over two steps (Scheme 1A). Similarly, L-Cit was combined with aKG, $\mathrm{FeSO}_{4}$, asc and lysate of E. coliBL21(DE3) expressing Getl. Optimization of lysis conditions and head-space volume proved vital for obtaining complete conversion consistently on gram scale with $20 \mathrm{mM}$ substrate concentration (Table SI2). Ion-exchange purification, Boc and TBS protections, followed by methyl esterification resulted in a $41 \%$ yield of 10 over four steps (Scheme 1B). Due to the acid labile nature of the $2^{\circ}$ TBS group, removal of the Boc group necessitated the use of TESOTf buffered by 2,6-lutidine.10a,b These conditions presumably proceed through the intermediacy of a silyl carbamate, which is hydrolyzed upon work-up. The resulting amine 7 was carried on crude to peptide coupling with 6 , yielding 4 after saponification (Scheme 1C).

Scheme 1. A. GetF catalyzed hydroxylation of L-Pip and subsequent Boc protection. B. Getl catalyzed hydroxylation of L-Cit and subsequent protecting group manipulations. C. Peptide coupling between 6 and 7 followed by saponification.

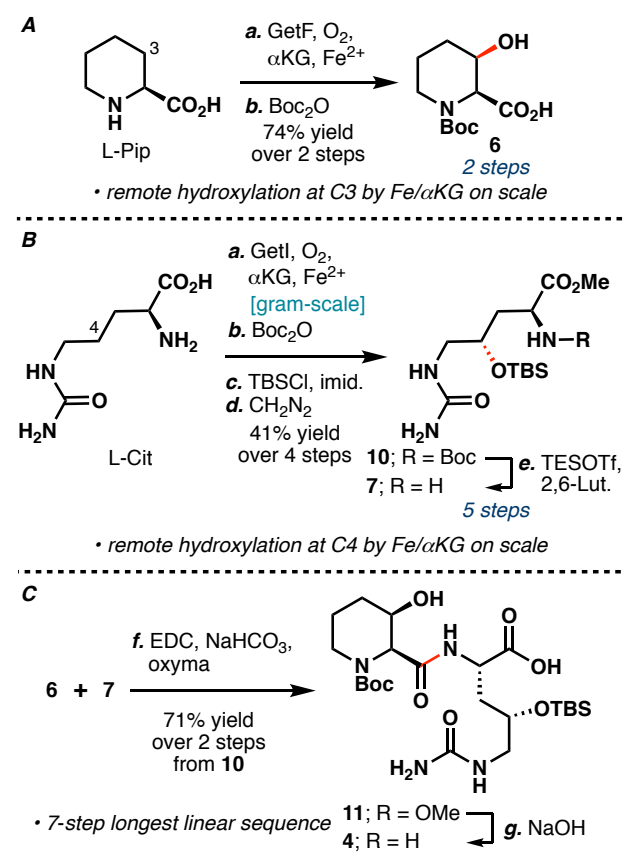

With access to 4 , construction of dipeptide 5 could now commence (Scheme 2). Initially, we tested previously reported conditions for 2-azotisation of BocHis-OMe ( $\mathrm{HCl}, \mathrm{NaNO}_{2}, \mathrm{pH}=10.5 \mathrm{Na}_{2} \mathrm{~B}_{4} \mathrm{O}_{7}$ buffer, 4aminobenzoate, $65 \%$ ), which unfortunately gave $6 \%$ yield in our hands. ${ }^{7}$ After testing a variety of azo coupling conditions (Table SI3), we eventually discovered that modulating the initially tested conditions by switching the coupling partner (4-aminobenzoate to 4-bromoaniline) gave $48 \%$ yield of our desired 2 azoimidazole (Scheme 2A). Saponification of the methyl ester gave acid $\mathbf{8}$, which was used crude in subsequent peptide coupling. To construct AA4, we initially attempted Myers' asymmetric aldol using pseudophenamine glycinamide as a chiral auxiliary, but the resulting aldol adduct proved unstable to purification or subsequent transformations; in-line with their previous report. ${ }^{11}$ As an alternative, we investigated $\mathrm{TiCl}_{4}$-mediated aldol conditions previously reported by Franck for the synthesis of syn-1,2-azidoalcohols from azidoacetyl-4-phenylthiazolidin-2-thione (13, Scheme 2B). ${ }^{12}$ To our dismay, 2-chloro-5formyl-BOM-imidazole (14) showed only minor product by LC-MS analysis, and no observable product 
upon work-up (Scheme 2, blue inset). Switching the BOM protecting group to Trt (15) showed no reaction by LC/MS or NMR. Based on these findings, we speculated that increased steric congestion proximal to the aldehyde might block the titanium enolate's approach. In line with this hypothesis, 4formyl-Trt-imidazole (16), which places the Trt protecting group distal to the aldehyde gave $7: 1 \mathrm{dr}$ and $33 \%$ isolated yield after methanolysis. The use of 2chloro-4-formyl-Trt-imidazole (12, accessible from known SI4 in one step) in the reaction gave the syn1,2 -azido-alcohol product in $59 \%$ yield as a single diastereomer on gram-scale. Subsequently, azide reduction proved non-trivial, resulting in trityl deprotection, chloride reduction, or non-specific degradation under the conditions tested (Table SI4). A report by Boger used gaseous hydrogen sulfide $\left(\mathrm{H}_{2} \mathrm{~S}\right)$ for azide reduction in the preparation of related histidine analogs. ${ }^{13}$ As this reagent is toxic and not widely available, we instead tried aqueous ammonium sulfide $\left(\left(\mathrm{NH}_{4}\right)_{2} \mathrm{~S}\right)$, which very cleanly yielded the desired amino alcohol 9 without the need for subsequent purification (Table SI4, entry 9). ${ }^{14}$ To the best of our knowledge, this is the first reported use of these conditions for the reduction of alkyl azides.

Scheme 2. A. Azo coupling on Boc-L-His-OMe to prepare the masked 2-aminohistidine motif and subsequent hydrolysis. B. Diastereoselective synthesis of 9 via Franck aldol between 12 and 13 followed by azide reduction. C. Synthesis of key dipeptide 5 by peptide coupling between 8 and 9 followed by Boc deprotection. Blue inset: substrate preference of Franck aldol with 13. Teal inset: Model study for chemoselective reduction of the 2-azoimidazole moiety.
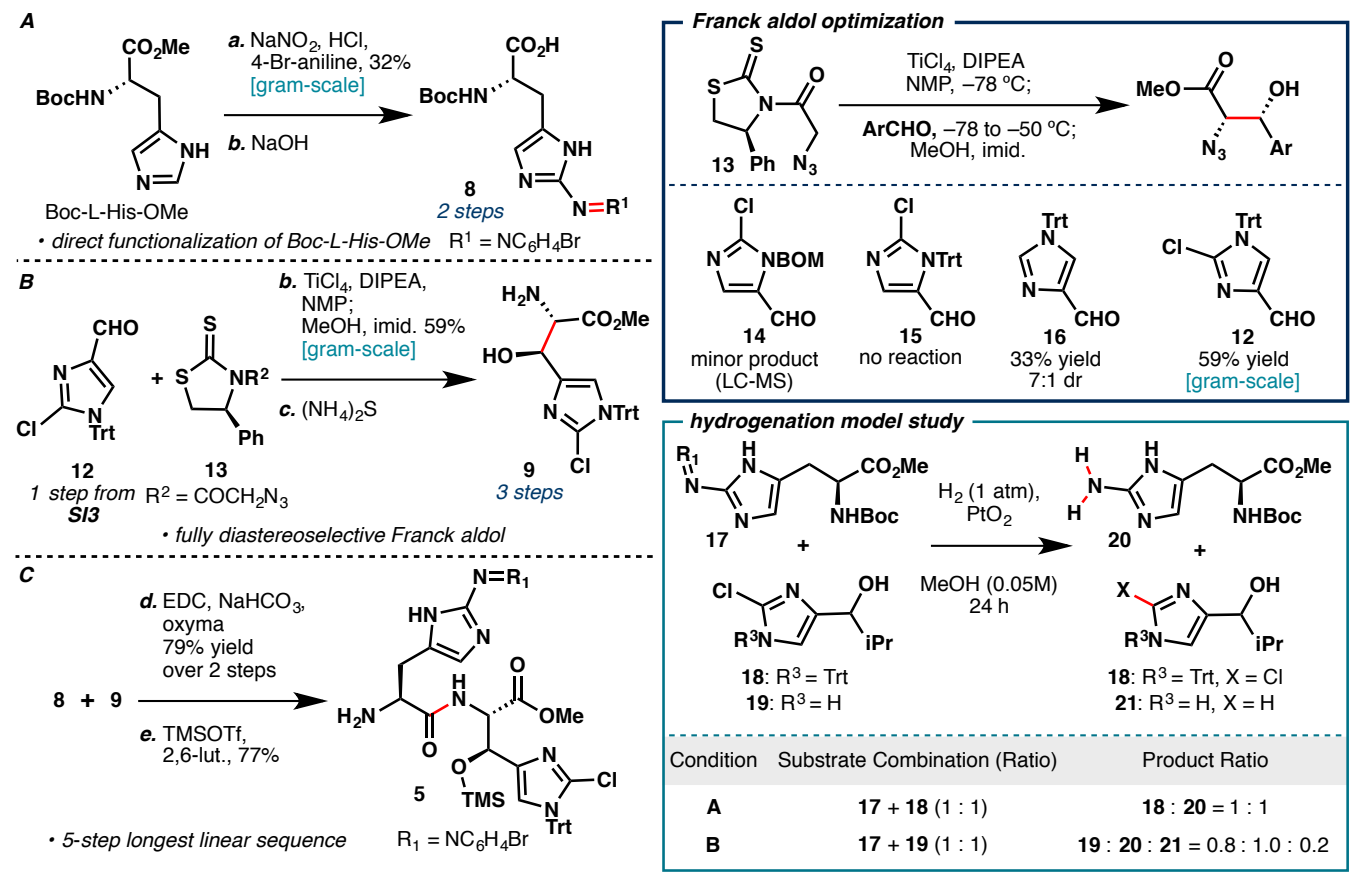

Since late-stage chloride reduction was of concern, we designed a model system to probe the viability of performing a chemoselective 2-azoimidazole hydrogenation in the presence of a 2-chloroimidazole-containing molecule (Scheme 2, teal inset).
Submitting an equimolar ratio of 17 and AA4 surrogate 18 to reaction with $\mathrm{H}_{2}$ and $\mathrm{PtO}_{2}$ gratifyingly yielded chemoselective azo hydrogenation. ${ }^{7}$ To confirm that chemoselectivity was imparted by the Trt, we also submitted an equimolar ratio of 17 and des- 
Trt-alcohol 19 to the same hydrogenation conditions, which gave a $4: 1$ mixture of 19 to des-chloro 21. These observations suggest that Trt protection imparts chemoselectivity between the azo and chloro functionality and that control of the steric environment around the 2-chloroimidazole would allow for late-stage removal of the azo functionality en route to GE81112 B1.

With conditions for late-stage azo removal in hand, we next targeted dipeptide 5 (Scheme 2C). Careful temperature control (e.g. slow warm from $-40^{\circ} \mathrm{C}$ to rt) proved necessary to avoid epimerization when coupling 8 and $\mathbf{9}$. Under these conditions, diasteriomerically pure product could be obtained in $79 \%$ over two steps. ${ }^{4}$ Similar to 10 , Boc deprotection required exceptionally mild conditions to avoid Trt deprotection, therefore we tested silyltriflates buffered by 2,6-lutidine (Table SI5). While TBSOTf and TESOTf resulted in stable TBS carbamate and Trt deprotection respectively, TMSOTf cleanly afforded 5 in $77 \%$ isolated yield. ${ }^{10}$ With fragments 4 and 5 in hand, we proceeded to construct the core tetrapeptide of GE81112 B1 (Scheme 3). Slow addition of 4 to a solution of 5, EDC, Oxyma and $\mathrm{NaHCO}_{3}$ cleanly afforded 22 as a single diastereomer in $66 \%$ isolated yield. ${ }^{4}$ In line with our model hydrogenation, 22 was chemoselectively reduced to amine 23 in $66 \%$ yield under the action of $\mathrm{H}_{2}$ and $\mathrm{PtO}_{2}$. Subsequent methyl ester hydrolysis followed by global deprotection and HPLC purification yielded GE81112 B1 in 36\% over two steps as the TFA salt.

Scheme 3. Peptide coupling of 4 and 5 followed by deprotection sequence to complete the synthesis of GE81112 B1.

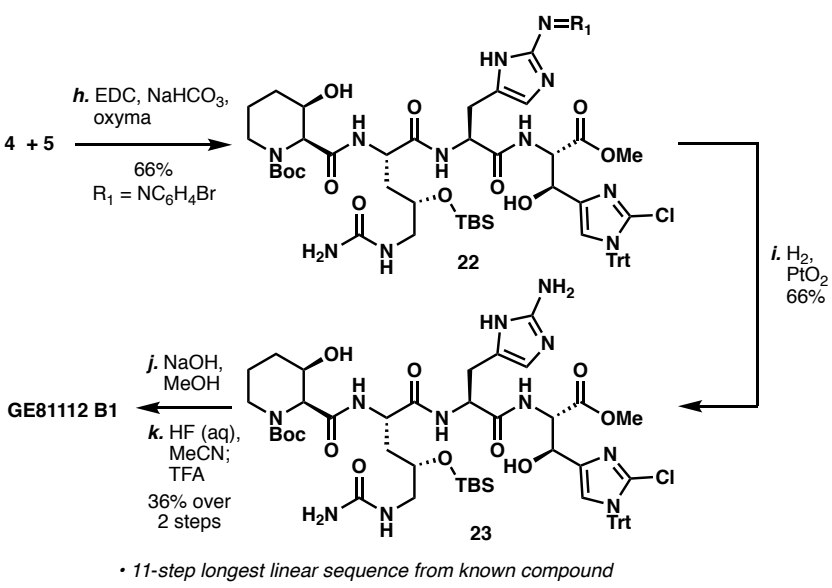

Having established a tractable route to GE81112 B1 (3), we set out to determine its key pharmacophores that significantly contribute to its antimicrobial activity. Prior effort by Fabbretti et al. has resulted in a solved crystal structure of 3:30S complex. ${ }^{2 b}$ However, we noted a lack of electron density around the ligand in this structure, which likely renders it unreliable for structure-based analog design. Conversely, SAR investigation between 3 and its antimicrobial activity would shed light into the contribution of each structural motif to the inhibitory activity of the tetrapeptide and aid future efforts in designing superior analog(s) or simplified analog(s) with comparable activity. We were especially interested in assessing the importance of the five peripheral modifications for inhibitory activity (Scheme 4). To this end, we prepared five simplified analogs of 3 (24 28), each containing one less peripheral modification than the parent natural product. Synthesis of analogs 24,25 and 26 proceeded in a routine fashion, requiring the use of readily available building blocks Boc-L-Pip-OH, L-Cit-OMe and Boc-L-His-OMe respectively in a route that is almost identical to Schemes 1-3.

Previously, we reported the synthesis of Cl-L-HisOMe 32 for functional characterization of Getl.6c While useful for analytical scale preparation of $\mathbf{3 2}$, 
our reported approach suffers from low yield and limited scalability. In turn, preparation of analog 27 necessitated the development of a novel strategy that remedies these synthetic shortcomings towards 2-chloro-L-histidine. Direct chlorination of Boc-LHis-OMe gave only $C 4$ chlorination using $S_{E} A r$ conditions (NCS or PIDA/NaCl) or the ketone following lithiation (Figure SI4). ${ }^{15}$ An effective ANRORC reaction with phenyl chloroformate afforded oxo-Boc-LHis-OMe (SI29) in 85\% yield, although only minor amounts of $\mathbf{3 2}$ were observed while testing various chlorination conditions. ${ }^{16 a}$ Success was finally achieved by developing an analogous ANRORC reaction utilizing phenyl thionochloroformate (29) to produce thio-Boc-L-His-OMe 30 in $90 \%$ yield. ${ }^{16 b}$ Chemoselective oxidation of $\mathbf{3 0}$ with NCS afforded the intermediate sulfonyl chloride (31), which was then converted to $\mathbf{3 2}$ following desulfination under gentle heating. 17 a This novel reaction sequence proceeds on $>500 \mathrm{mg}$ scale without any observable loss in isolated yield, solving the key limitations observed in our previous route. The unique desulfinative chlorination from $\mathbf{3 1}$ to $\mathbf{3 2}$ may proceed via the intermediacy of a chlorine radical that participates in ipso substitution of the sulfonyl chloride, regenerating chlorine radical following sulfur dioxide extrusion. ${ }^{17 b}$ Although the exact precursor to chlorine radical is puzzling, this mechanism seems operative, given many analogous literature reactions that proceed under standard radical chlorination conditions. ${ }^{17 b, c}$ Another possibility involves concerted homolysis of the $\mathrm{C}-\mathrm{S}$ and $\mathrm{S}-\mathrm{Cl}$ bonds in 31 , followed by recombination of the resulting carbon and chlorine radicals to $32.17 \mathrm{a}, \mathrm{d}$ However, this mechanism seems less likely given the high bond dissociation energy (BDE) of $\mathrm{C}-\mathrm{S}$ and $\mathrm{S}-\mathrm{Cl}$ bonds ( 67 $\mathrm{kcal} / \mathrm{mol}) .{ }^{17 \mathrm{~d}}$

Further difficulty in the synthesis of $\mathbf{2 7}$ was experienced when the trityl-protected derivative of 32 proved to be more labile than $\mathbf{9}$, posing significant challenge in selective Boc deprotection after coupling with 8. As a workaround, linear assembly to a tripeptide intermediate consisting of 6,7 and 8 was first prepared and submitted to a final peptide coupling with SI32, which provided analog 27 after deprotection. Our synthesis of 27 delineates an alternative assembly strategy to the GE81112 core that is additionally more well-suited to diversity-oriented synthesis in the production of analogs. Finally, the use of a Franck aldol on aldehyde 16 and selective azide reduction provided protected $\beta$-hydroxy-Lhistidine 33, albeit in diminished yield and diastereoselectivity. Nevertheless, 33 could be submitted to the same synthetic sequence described in Schemes 2 and 3 to afford analog 28.

Scheme 4. Synthesis of simplified analogs 24-28 and a 3-OH-Pro-containing analog (35), and their MIC measurements enabled initial SAR exploration of GE81112 B1 and elucidation of its key pharmacophores. MIC values are provided as a range obtained from three measurements. 

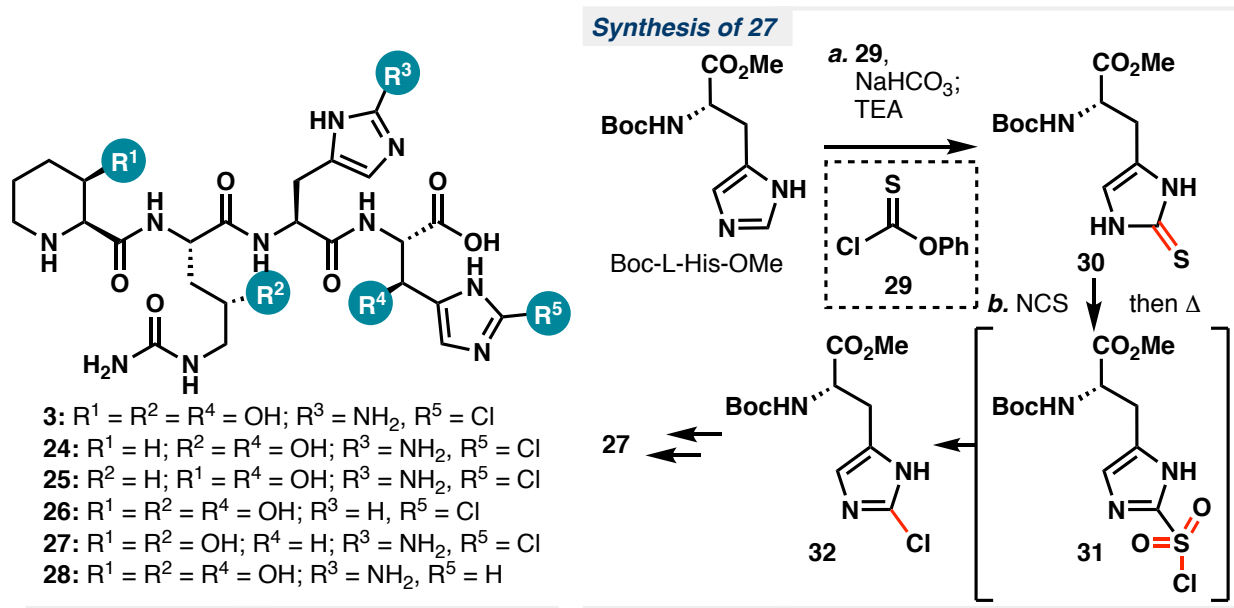

3: $\mathrm{R}^{1}=\mathrm{R}^{2}=\mathrm{R}^{4}=\mathrm{OH} ; \mathrm{R}^{3}=\mathrm{NH}_{2}, \mathrm{R}^{5}=\mathrm{Cl}$ 24: $\mathrm{R}^{1}=\mathrm{H} ; \mathrm{R}^{2}=\mathrm{R}^{4}=\mathrm{OH} ; \mathrm{R}^{3}=\mathrm{NH}_{2}, \mathrm{R}^{5}=\mathrm{Cl}$ 25: $R^{2}=H ; R^{1}=R^{4}=O H ; R^{3}=N_{2}, R^{5}=C l$ 26: $R^{1}=R^{2}=R^{4}=O H ; R^{3}=H, R^{5}=C l$ 27: $R^{1}=R^{2}=\mathrm{OH} ; R^{4}=H ; R^{3}=N_{2}, R^{5}=C$ 28: $R^{1}=R^{2}=R^{4}=O H ; R^{3}=N_{2}, R^{5}=H$

Synthesis of 24-26

Boc-L-Pip-OH

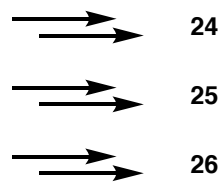

Boc-L-His-OMe

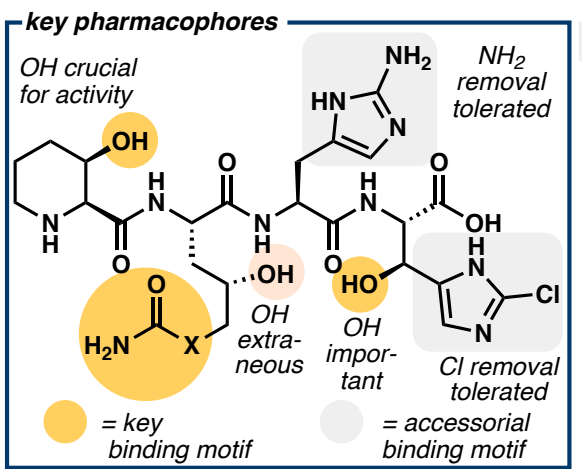

Synthesis of 28

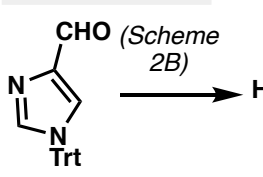

16

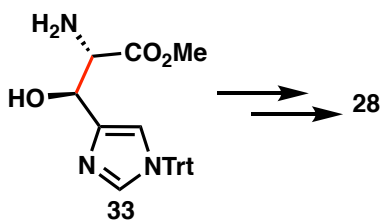

Synthesis of $35 \quad 34$ available commercially
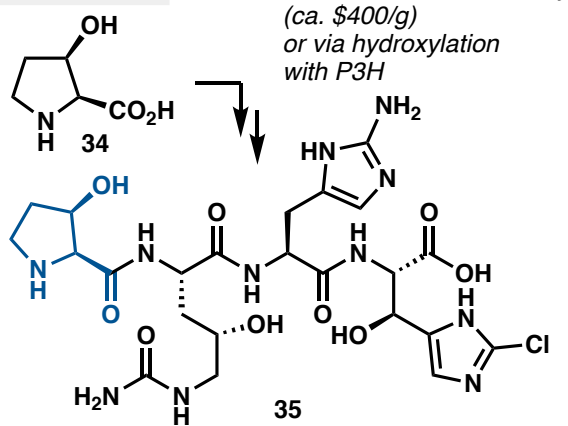

\begin{tabular}{c|ccccccc} 
Compound & $\mathbf{3}$ & $\mathbf{2 4}$ & $\mathbf{2 5}$ & $\mathbf{2 6}$ & $\mathbf{2 7}$ & $\mathbf{2 8}$ & $\mathbf{3 5}$ \\
\hline MIC $(\mu \mathrm{g} / \mathrm{mL}$, & $<0.0039$ & $>256$ & $<0.0039$ & 0.063 & $2-4$ & 0.016 & $<0.0039$ \\
E. coli MG1655) & & & &
\end{tabular}

Analogs 24-28 were next subjected to antibacterial assays, consisting of minimal inhibitory concentration (MIC) measurements against $E$. coli strain MG1655 in minimal media with $\mathbf{3}$ and gentamycin as a control. The MIC obtained for $\mathbf{3}$ was in close agreement with previous data in the literature. ${ }^{1,3,4}$ Analog 24 was found to be completely inactive. In conjunction with previous finding from Sanofi, this result suggests that the syn- $\beta$-hydroxy amino acid unit in AA1 is highly crucial for inhibitory activity. ${ }^{4}$ Analog 25 , containing unmodified L-Cit at AA2 was found to maintain the antibacterial activity of the parent natural product. This finding points to the extraneous nature of the $\gamma-\mathrm{OH}$ unit in AA2 for activity and also the possibility of further developing simplified analog(s) of 3 with equipotent antibacterial activity. Removal of the $\mathrm{C} 2-\mathrm{NH}_{2}$ moiety in $\mathrm{AA} 3$ or $\mathrm{C} 2-\mathrm{Cl}$ in $\mathrm{AA} 4$ only reduced the activity of the resulting compounds by $5-15$ fold, while removal of the $\beta-O H$ in $\mathrm{AA} 4$ led to a more than 500-fold reduction in activity. These data show that the $\beta-\mathrm{OH}$ motif is the most crucial peripheral modification within the AA3-AA4 dipeptide for antibacterial activity.

Taken together, we obtained the first preliminary SAR for the antibacterial activity of GE81112 B1. Based on our data, we posit that the AA1-AA2 dipeptide, in conjunction with the $\beta-\mathrm{OH}$ on $\mathrm{AA} 4$, 
constitutes the primary binding motif of the tetrapeptide while the peripherally modified imidazole sidechains of $A A 3$ and $A A 4$ act only as accessorial binding motifs to fine-tune the binding affinity of the molecule. Based on the slightly superior antibacterial profile of GE81112 B (2), we presume that a ureido or a carbamate motif on AA2 is capable of eliciting favorable polar or hydrogen bonding interactions in the binding pocket and that the difference in activity between 2 and 3 arises from the hydrogen-bonding donor/acceptor property of the internal nitrogen/oxygen at their AA2's $\delta$-carbon. The importance of the syn- $\beta$-hydroxy amino acid unit in AA1 begged the question whether this property is highly specific to 3hydroxy-L-pipecolic acid or whether substitution with other cyclic syn- $\beta$-hydroxy amino acids is tolerated at this position. To this end, we synthesized an analog containing 3-hydroxy-L-proline at AA1 from 34, which is commercially available at ca. $\$ 400 / g$ or can be prepared via biocatalytic hydroxylation with the Fe/ $\alpha$ KG enzyme P3H.18a,b,c,d Gratifyingly, analog 35 was found to be equipotent to 3 . This result suggests tolerance to alternative ring sizes at $\mathrm{AA} 1$ and perhaps more importantly, the potential of developing further analogs of 3 by enlisting the use of other cyclic syn- $\beta$-hydroxy amino acids at AA1. The latter can be readily achieved in a biocatalytic fashion using several known Fe/ $\alpha \mathrm{KG}$ cyclic amino acid hydroxylases. ${ }^{6 b, 18}$ Thus, while the synthesis and antibacterial assay of $\mathbf{3 5}$ provides an initial singular case, it lays the groundwork for using a pool of Fe/aKG hydroxylases in a medicinal chemistry setting to rapidly perform SAR studies on a lead molecule to identify superior analogs.

In conclusion, this work describes the development of a chemoenzymatic strategy to arrive at the first synthesis of GE81112 B1, and the application of this strategy for the synthesis of six analogs. By combining biocatalytic hydroxylation and traditional chemical methods, we developed a strategy that requires only $2-5$ steps ( 3 steps average) to synthesize each AA fragment. Additionally, our hybrid approach allows each key fragment to be prepared with high levels of stereocontrol. Thus, our strategy not only constitutes a distinct departure from contemporary approaches to morphed peptide synthesis, but also provides a robust solution to some of the shortcomings encountered in previous synthetic studies on GE81112 A. ${ }^{4}$ Similar to our previous work, 8,9,19 this report highlights the strategic benefits of using enzymatic $\mathrm{C}-\mathrm{H}$ oxidation to streamline access to complex, bioactive natural products. Our efficient strategy to access GE81112 B1 also enabled the preparation of six analogs, which in turn allowed us to elucidate the key pharmacophores of GE81112 B1 and obtain the first SAR insights into its inhibitory activity. This work also provides an initial validation for using other Fe/ $\alpha \mathrm{KG}$ amino acid hydroxylases in generating unnatural analogs of GE81112 B1 for further medicinal chemistry optimization. Studies in this area are ongoing in our laboratory and will be reported in due course.

\section{ASSOCIATED CONTENT}

Experimental details, analytical data, ${ }^{1} \mathrm{H}$ and ${ }^{13} \mathrm{C}$ NMR data.

\section{AUTHOR INFORMATION}

Corresponding Author

hrenata@scripps.edu.

\section{ORCID}

Hans Renata: 0000-0003-2468-2328

Notes

The authors declare no competing financial interest.

\section{ACKNOWLEDGMENT}


This work is supported, in part, by the National Institutes of Health Grant GM128895 (H.R.). We thank Prof. Ian Seiple for useful discussions on asymmetric aldol reactions. We thank Drs. Andrew Steele and Christiana Teijaro for providing assistance with MIC measurements. We acknowledge the Roush, Bannister and Shen labs for generous access to their reagents and instrumentation.

\section{REFERENCES}

1. Brandi, L.; Lazzarini, A.; Cavaletti, L.; Abbondi, M.; Corti, E.; Ciciliato, I.; Gastaldo, L.; Marazzi, A.; Feroggio, M.; Fabbretti, A.; Maio, L.; Colombo, L.; Donadio, S.; Marinelli, F.; Losi, D.; Gualerzi, C. O.; Selva, E. Novel Tetrapeptide Inhibitors of Bacterial Protein Synthesis Produced by a Streptomyces sp. Biochemistry 2006, 45, 3692-3702.

2. a) Brandi, L.; Abbondi, M.; Fabbretti, A.; Donadio, S.; Losi, D.; Gualerzi, C. O. Specific, efficient, and selective inhibition of prokaryotic translation initiation by a novel peptide antibiotic. Proc. Natl. Acad. Sci. 2006, 103, 39-44. b) Fabbretti, A.; Andreas, S.; Brandi, L.; Kaminishi, T.; Giuliodori, A. M.; Garofalo, R.; Ochoa-Lizarralde, B.; Takemoto, C.; Yokoyama, S.; Connell, S. R.; Gualerzi, C. O.; Fucini, P. Inhibition of translation initiation complex formation by GE81112 unravels a 16S rRNA structural switch involved in P-site decoding. Proc. Natl. Acad. Sci. 2016, 113, E2286-E2295. c) López-Alonso, J. P.; Fabbretti, A.; Kaminishi, T.; Iturrioz, I.; Brandi, L.; Gil-Carton, D.; Gualerzi, C. O.; Fucini, P.; Connell, S. R. Structure of a 30 S pre-initiation complex stalled by GE81112 reveals structural parallels in bacterial and eukaryotic protein synthesis initiation pathways. Nucleic Acids Research 2017, 45, 2179-2187.

3. Maio, A.; Brandi, L.; Donadio, S.; Gualerzi, C. O. The Oligopeptide Permease Opp Mediates Illicit Transport of the
Bacterial P-site Decoding Inhibitor GE81112. Antibiotics

2016, 5, 17 .

4. Jürjens, G.; Schuler, S. M. M.; Kurz, M.; Petit, S.; Couturier, C.; Jeannot, F.; Nguyen, F.; Wende, R. C.; Hammann, P. E.; Wilson, D. N.; Bacqué, E.; Pöverlein, C.; Bauer, A. Total Synthesis and Structural Revision of the Antibiotic Tetrapeptide GE81112. Angew. Chem. Int. Ed. 2018, 57, $12157-12161$

5. a) Turner, N. J.; O'Reilly, E. Biocatalytic retrosynthesis. Nat. Chem. Biol. 2013, 9, 285- 288. (b) de Souza, R. O. M. A.; Miranda, L. S. M.; Bornscheuer, U. T. A Retrosynthesis Approach for Biocatalysis in Organic Synthesis. Chem. -Eur. J. 2017, 23, 12040-12063.

6. a) Binz, T. M.; Maffioli, S. I.; Sosio, M.; Donadio, S.; Müller, R. Insights into an Unusual Nonribosomal Peptide Synthetase Biosynthesis Identification and Characterization of the GE81112 Biosynthetic Gene Cluster. J Biol Chem. 2010, 285, 32710-32719. b) Mattay, J.; Hüttel, W. Pipcolic Acid Hydroxylases: A Monophyletic Clade among cis-Selective Bacterial Proline Hydroxylases that Discriminates L-Proline. ChemBioChem 2017, 18, 1523-1528. c) Zwick, C. R. III; Sosa, M. B.; Renata, H. Bioinformatics-Guided Discovery of Citrulline 4-Hydroxyalse from GE81112 Biosynthesis and Rational Engineering of Its Substrate Specificity. Angew. Chem. Int. Ed. 2019, 58, 18854-18858.

7. Jaganathen, A.; Ehret-Sabatier, L.; Bouchet, M.-J.; Goeldner, M. P. Synthesis and Properties of a 2-Diazohistidine Derivative: A New Photoactivatable Aromatic Amino-Acid Analog. Helvetica Chimica Acta 1990, 73, 86-96.

8. Zhang, X.; Renata, H. Efficient chemoenzymatic synthesis of (2S,3R)-3-hydroxy-3-methylproline, a key fragment in 
polyoxypeptin A and FR225659. Tetrahedron 2019, 75, 3253-3257.

9. a) Zhang, X.; King-Smith, E.; Renata, H. Total Synthesis of Tambromycin by Combining Chemocatalytic and BiocataIytic C-H Functionalization. Angew. Chem. Int. Ed. 2018, 57, 5037-5041. b) Thomas, J. G.; Ayling, A.; Baneyx, F. Molecular chaperones, folding catalysts, and the recovery of active recombinant proteins from E. coli. To fold or to refold. Appl. Biochem. Biotechnol. 1997, 66, 197-238.

10. a) Sakaitani, M.; Ohfune, Y. Selective Transformation of Nt-Butoxycarbonyl Group into N-Alkoxy-Carbonyl Group via N-Carboxylate Ion Equivalent. Tet. Lett. 1985, 26, 55435546. b) Zhang, Y.-H.; Liu, R.; Liu, B. Total synthesis of nannocystin Ax. Chem. Commun. 2017, 53, 5549-5552. c) Li, P.; Evans, C. D.; Wu, Y.; Cao, B.; Hamel, E.; Joullié, M. M. Evolution of the Total Synthesis of Ustiloxin Natural Products and Their Analogues. J. Am. Chem. Soc. 2008, 130, 2351-2364. d). Nuhant, P.; Roush, W. R. Enantio- and Diastereoselective Synthesis of $\mathrm{N}$-Acetyl Dihydrotetrafibricin Methyl Ester. J. Am. Chem. Soc. 2013, 135, 5340-5343.

11. Seiple, I. B.; Mercer, J. A. M.; Sussman, R. J.; Zhang, Z.; Myers, A. G. Stereocontrolled synthesis of syn- $\alpha-H y d r o x y-$ $\beta$-amino acids by direct aldolization of pseudoephenamine glycinamide. Angew. Chem. Int. Ed. 2014, 53, 4642-4647.

12. Patel, J.; Clavé, G.; Renard, P.-Y.; Franck, X. Straightforward Access to Protected syn $\alpha$-Amino- $\beta$-hydroxy Acid Derivatives. Angew. Chem. Int. Ed. 2008, 47, 4224-4227.

13. Boger, D. L.; Colletti, S. L.; Honda, T.; Menezes, R. F. Total Synthesis of Bleomycin A2 and Related Agents. 1. Synthesis and DNA Binding Properties of the Extended C-
Terminus: Tripeptide S, Tetrapeptide S, Pentapeptide S, and Related Agents. J. Am. Chem. Soc. 1994, 116, 56075618

14. Lubriks, D.; Sokolovs, I.; Suna, E. Indirect C-H Azidation of Heterocycles via Copper-Catalyzed Regioselective Fragmentation of Unsymmetrical l3-lodanes. J. Am. Chem. Soc. 2012, 134, 15436-15442.

15. a) Jain, R.; Avramovitch, B.; Cohen, L. A. Synthesis of RingHalogenated Histidines and Histamines. Tetrahedron 1998, 54, 3235-3242. b) Himabundu, V.; Parvathaneni, S. P.; Rao, V. J. Phl(OAc)2/NaX-Mediated Halogenation Prodiving Access to Valuable Synthons 3-Haloindole Derivatives. New J. Chem. 2018, 42, 18889-18893.

16. a) Adam, W.; Zhao, C.-G.; Jakka, K. Dioxirane Oxidations of Compounds Other Than Alkenes. Org. React. 2007, 1346. b) Xu, J.; Yadan, J. C. Synthesis of L-(+)-Ergothioneine. J. Org. Chem. 1995, 60, 6296-6301.

17. a) Ford, M. E.; Dixon, D. D. Novel Chlorination of the 1,3,4Thiadiazole Ring. J. Heterocycl. Chem. 1980, 17, 13111312. b) Miller, B.; Walling, C. The Displacement of Aromatic Substituents by Halogen Atoms. J. Am. Chem. Soc. 1957, 79, 4187-4191. c) Traynham, J. G. Ipso Substitution in Free-Radical Aromatic Substitution Reactions. Chem. Rev. 1979, 79, 323-330. d) Navrotskii, A. V.; Stepanov, G. V.; Safronov, S. A.; Gaidadin, A. N.; Seleznev, A. A.; Navrostskii, V. A. Novakov, I. A. Homolytic Decomposition of Sulfonyl Chlorides. Doklady Chemistry 2018, 480, 93-95.

18. a) Mori, H.; Shibasaki, T.; Yano, K.; Ozaki, A. Purification and Cloning of a Proline 3-Hydroxylase, a Novel Enzyme Which Hydroxylastes Free L-Proline to cis-3-Hydroxy-LProline. J. Bacteriol. 1997, 179, 5677-5683. b) Shibasaki, 
T.; Sakurai, W.; Hasegawa, A.; Uosaki, Y.; Mori, H.; Yoshida, M.; Ozaki, A. Tetrahedron Lett. 1999, 40, 5227-5230. c) Shibasaki, T.; Mori, H.; Ozaki, A. Cloning of an Isozyme of Proline-3-Hydroxylase and Its Purification from Recombinant Escherichia coli. Biotechnol. Lett. 2000, 22, 19671973. d) Johnston, R. M.; Chu, L. N.; Liu, M.; Goldberg, S. L.; Goswami, A.; Patel, R. N. Hydroxylation of L-Proline to cis-3-Hydroxy-L-Proline by Recombinant Escherichia coli Expressing a Synthetic L-Proline-3-Hydroxylase Gene. Enzyme Microb. Technol. 2009, 45, 484-490. e) Du, Y.; Wang, Y.; Huang, T.; Tao, M.; Deng, Z.; Lin, S. Identification and Characterization of the Biosynthetic Gene Cluster of Polyoxypeptin A, a Potent Apoptosis Inducer. BMC Microbiol.

2014, 14, 30.
19. a) Zwick, C. R. III; Renata, H. Remote C-H Hydroxylation by an $\alpha$-Ketoglutarate-Dependent Dioxygenase Enables Efficient Chemoenzymatic Synthesis of Manzacidin C and Proline Analogs. J. Am. Chem. Soc. 2018, 140, 1165-1169.

b) Zwick, C. R. III; Renata, H. Evolution of Biocatalytic and Chemocatalytic C-H Functionalization Strategy in the Synthesis of Manzacidin C. J. Org. Chem. 2018, 83, 74077415. c) Zwick, C. R. III; Renata, H. A One-Pot Chemoen-

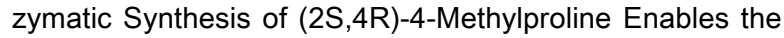
First Total Synthesis of Antiviral Lipopeptide Cavinafungin B. Tetrahedron 2018, 74, 6469-6473. d) Amatuni, A.; Renata, $H$. Identification of a lysine 4-hydroxylase from the glidobactin biosynthesis and evaluation of its biocatalytic potential. Biomol. Chem. 2019, 17, 1736-1739.

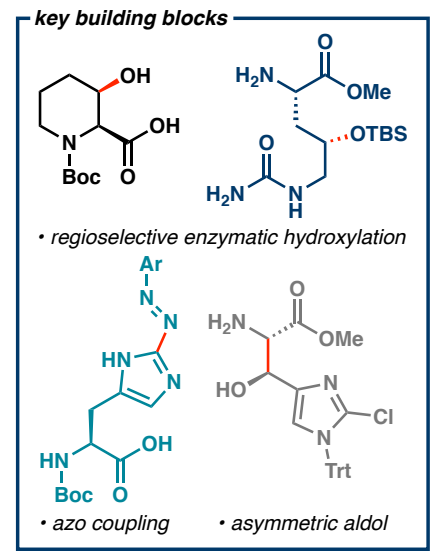

